\title{
Meson decay studies from MAMI A2
}

\author{
Lena Heijkenskjöld ${ }^{1, *}$ for the A2 collaboration \\ ${ }^{1}$ Institut für Kernphysik, Johannes Gutenberg-Universität Mainz, D-55099 Mainz, Germany
}

\begin{abstract}
Decays of the light mesons $\pi^{0}, \eta, \omega$, and $\eta^{\prime}$ provide a unique laboratory to test fundamental aspects of hadron physics. Precision studies of such diverse topics as the light quark mass ratio, $\pi-\pi$ scattering lengths, and searches for physics beyond the Standard Model are possible. Additionally, Dalitz decays of light mesons provide a way of measuring the electromagnetic meson transition form factors in the time-like region.

The A2 tagged photon facility at the Mainz Microtron provides a high yield of light mesons produced by photo-induced reactions on protons, which makes the experiment ideal for high precision measurements of meson decays. Presented here are the contributions made by the A2 collaboration to such studies.
\end{abstract}

\section{Introduction}

Studies of the interactions and properties of light mesons, $\pi^{0}, \eta, \omega$, and $\eta^{\prime}$, provide insight to fundamental aspects of hadron physics. They probe low-energy, and hence non-perturbative, QCD where models and effective field theories are used. These often require experimental measurements as input or for benchmarking. This text will discuss some of the important decay studies performed at the A2 experiment and the role they play in our understanding of low-energy QCD. The text is divided into two topics, one showing published and ongoing studies of meson transition form factors and one showing recent results from the A2 collaboration of Dalitz plot measurements.

\subsection{Meson Transition Form Factors}

Meson transition form factors (TFFs) describe the interaction between mesons and two real or virtual photons. Hence, they provide a unique tool to study the electromagnetic structure of the mesons. TFFs of light pseudoscalar mesons also play a significant role for the high precision frontier of the Standard Model (SM), more specifically for increasing the precision of the SM calculations of the anomalous magnetic moment of the muon, $a_{\mu}$. Currently there is a 3-4 $\sigma$ discrepancy between the experimentally measured value of $a_{\mu}$ [1] and the SM prediction [2]. With ongoing efforts to further reduce the experimental error [3, 4], efforts are also made on theory side to reduce the error of $a_{\mu}^{S M}$. The largest contribution to the error comes from the strong sector, where data-driven approaches need input from experimental measurements of, among others, TFFs of pseudoscalar mesons [5].

Experimentally, TFFs can be accessed in different ways for different virtualities of the photons. At the A2 experiment, the decays of the mesons are used to access the TFFs and,

\footnotetext{
*e-mail: lheijken@uni-mainz.de
} 
hence, the photons are time-like. For $\pi^{0}, \eta$ and $\eta^{\prime}(P)$, it is the single Dalitz decay, $P \rightarrow e^{+} e^{-} \gamma$, which has been studied here, while for $\omega$ it is $\omega \rightarrow e^{+} e^{-} \pi^{0}$. The TFF is extracted from the dependence of the decay rate on the momentum transfer of the virtual photon $q^{2}$ [6], typically normalised to the on-shell process,

$$
\frac{d \Gamma\left(A \rightarrow B \ell^{+} \ell^{-}\right)}{d q^{2} \Gamma(A \rightarrow B \gamma)}=[Q E D]\left|F_{A B}\left(q^{2}\right)\right|^{2} .
$$

As seen in (1), this spectrum is directly given by one part calculable from QED, which describes the point-like interaction, and the squared TFF of the process.

To facilitate comparisons between different TFF distributions, one often uses an Vector Meson Dominance (VDM) inspired one-pole approximation [6],

$$
F\left(q^{2}\right)=\frac{\Lambda_{V}^{2}}{\Lambda_{V}^{2}-q^{2}-i \Gamma_{V} \Lambda_{V}} \stackrel{q^{2}<\Lambda_{V}}{\approx} 1+\Lambda_{V}^{-2} q^{2}
$$

\subsection{Dalitz plot studies}

Dalitz plots allow for a direct access to the dynamics of three body decays, as the density distribution is given by the amplitude, $\mathcal{A}$, of the decay. For the recent Dalitz plot results from A2 of $\eta \rightarrow \pi^{0} \pi^{0} \pi^{0}$ and $\eta^{\prime} \rightarrow \eta \pi^{0} \pi^{0}$, it is particularly the rescattering effects which are of interest. The $\eta \rightarrow 3 \pi$ decay channels provide the possibility to access the mass ratio of the lightest quarks. However, $\mathcal{A}$ is strongly affected by rescattering effects which have to be carefully modeled [7]. Measurements of the $\eta^{\prime} \rightarrow \eta \pi \pi$ Dalitz plot provide a probe of Chiral Pertubation Theory (ChPT) expansions, like large- $N_{C}$ ChPT and Resonance Chiral Theory $[8,9]$, as well as a test of predictions made by dispersive relations [10].

Both decays have $\pi^{0} \pi^{0}$ in their final state, hence the effect of rescattering into a charged pion pair can be investigated. This effect is expected to cause a cusp in the $m_{\pi \pi}$ distribution at the threshold of the charged pair. This cusp was first observed by NA48/2 in the $K^{+} \rightarrow$ $\pi^{0} \pi^{0} \pi^{+}$decay. Several theoretical approaches have been used to model this rescattering effect and allowed to extract the S-wave $\pi \pi$ scattering length combination, $a_{0}-a_{2}$. Using a nonrelativistic effective field theory (NREFT) framework, this effect has been predicted be of $1 \%$ magnitude for $\eta \rightarrow \pi^{0} \pi^{0} \pi^{0}$ [11] and of $6 \%$ magnitude for $\eta^{\prime} \rightarrow \eta \pi^{0} \pi^{0}$ [12].

When constructing a Dalitz plot, where two of the final state masses are equal, a common choice of variables are the normalised $\mathrm{X}$ and $\mathrm{Y}$ variables constructed as

$$
X=\sqrt{3} \frac{T_{1}^{C M}-T_{2}^{C M}}{Q} \text { and } Y=\frac{\left(2 m_{1}+m_{3}\right) T_{3}^{C M}}{m_{1} Q}-1,
$$

where $T_{i}^{C M}$ are the kinetic energies of the final state particles in the centre of mass frame and $\mathrm{Q}$ is the total available kinetic energy. If one expects $\mathcal{A}$ to be completely symmetric under the exchange of the final state particles, e.g. as for $\eta \rightarrow 3 \pi^{0}$, one can instead use the normalised polar variables,

$$
z=X^{2}+Y^{2} \text { and } \phi=\arctan (Y / X) .
$$

If a smooth distribution is expected, the Dalitz plot can be parametrised using polynomial expansions,

$$
\begin{aligned}
|\mathcal{A}|^{2} & \propto N\left(1+a Y+b Y^{2}+c X+d X^{2}+\ldots\right) \\
|\mathcal{A}|^{2} & \propto N\left(1+2 \alpha z+2 \beta z^{2 / 3} \sin (3 \phi)+\ldots\right)
\end{aligned}
$$


For the $\eta \rightarrow 3 \pi^{0}$ Dalitz plot, the first parameter in this expansion, $\alpha$, has been wellmeasured by experiments and predicted by theory. In some models, also non-zero higher order parameters have been predicted, but never before measured. Previous measurements and predictions of the $\eta^{\prime} \rightarrow \eta \pi \pi$ Dalitz plot yielded discrepancies in the Dalitz plot parameters $a, b$ and $d$.

\section{Experimental Setup}

The A2 experimental facility is situated at the Mainz Microtron (MAMI) in Germany [13, 14]. MAMI provides a stable, near continuous, electron beam with maximum beam energy of $1604 \mathrm{MeV}$. In the A2 hall, this electron beam impinges on a radiator and produces a photon beam via the bremstrahlung process. The energy of the resulting photon beam is measured by diverting the scattered electrons and tagging them in either the Glasgow photon tagger [15] or the End Point Tagger (EPT) [16]. The resulting photon beam energies cover the production region of the lightest mesons, $\pi^{0}, \eta, \omega$ and, with EPT, $\eta^{\prime}$. The photon beam is collimated and then continues into the fixed-target CB-TAPS detector setup [17]. The target used for the studies presented here was a liquid hydrogen target of either $5 \mathrm{~cm}$ or $10 \mathrm{~cm}$ length. Directly surrounding the target is the Particle Identification Detector (PID), which is a barrel of 24 thin plastic scintillator paddles. PID is primarily used to veto charged particles. Surrounding the PID are two cylindrical multiwire proportional chambers (MWPCs). The MWPCs allow for charged particle detection and track reconstruction. Outside the MWPCs is the Crystal Ball (CB) detector, which is a spherically shaped calorimeter, consisting of $672 \mathrm{NaI}(\mathrm{Tl})$ crystals. $\mathrm{CB}$ covers the $\theta$ region between $20^{\circ}-160^{\circ}$. To further cover the forward direction, the Two Arm Photon Spectrometer (TAPS) is situated downstream, providing a coverage of the $\theta$ region from $20^{\circ}$ down to $2^{\circ}-4^{\circ}$. TAPS is a hexagonally shaped wall consisting of 366 $\mathrm{BaF}_{2}$ and $72 \mathrm{PbWO}_{4}$ crystals. Just in front of TAPS is a wall of 384 , thin, plastic scintillators, which can be used to detect charged particles.

\section{Results}

\subsection{Meson Transition Form Factors}

\subsection{1 $\pi^{0} \rightarrow e^{+} e^{-} \gamma$}

The A2 collaboration has published a high statistics result on the singly-virtual $\pi^{0}$ TFF based on the measurement of the $\pi^{0}$ Dalitz decay [18]. This study is based on two separate datasets, the first collected with an electron beam energy of $885 \mathrm{MeV}$ and the second with $1557 \mathrm{MeV}$. In the analysis, events with 3 or 4 clusters in the calorimeters are selected, allowing for unmeasured protons. A kinematic fit, requiring 4-momentum conservation, is applied. A resonant background contribution comes from the $\pi^{0} \rightarrow \gamma \gamma$ channel, where one of the photons converts into an $e^{+} e^{-}$pair. This can be suppressed by requiring the leptons to be detected in different PID elements. This comes at the expense of lower statistics, especially at small dilepton masses. The final event sample contains $4 \times 10^{5}$ Dalitz decay events used to produce the TFF distributions.

The QED contribution is calculated including radiative corrections [19] and the resulting data points from both datasets are provided with total errors. The parametrisation, given in (2), is used to extract the slope parameter $a_{\pi^{0}}=m_{\pi^{0}}^{2} \Lambda^{-2}=0.030 \pm 10_{\text {tot }}$. This result drastically increased the precision of $a_{\pi^{0}}$ extracted from direct measurement in the time-like momentum transfer region, only superseded by the results from NA62 [20]. 
During 2018, the A2 collaboration collected a large $\pi^{0}$ data set, which yielded a 5-6 times increase in the collected statistics of $\pi^{0}$ Dalitz decays. The main purpose of this data set is to produce a high statistics measurement of the $\pi^{0}$ TFF, which will provide world leading precision of the slope parameter from a direct time-like measurement.

\subsection{2 $\eta \rightarrow e^{+} e^{-} \gamma$}

The A2 collaboration has published several high statistics measurements of the singly-virtual time-like $\eta$ TFF [21-23] using $e^{+} e^{-}$pairs in the final state. These provide unprecedented and fully complementary contributions to the low dilepton mass region, compared to other high statistics measurements $[24,25]$ which used dimuons.

The most recent of the A2 studies is based on two datasets, the first one taken with an electron beam energy of $1508 \mathrm{MeV}$ and the second with $1557 \mathrm{MeV}$. The analysis strategy selects events with 3 or 4 clusters in the calorimeters, allowing for unmeasured protons. The kinematic fit procedure of requiring 4-momentum conservation is applied both to select the signal channel and to reject events which fits better to background hypotheses. The dileptons are identified using the PID, where cuts in the deposited energy allow for separation between leptons and protons. Requiring the leptons to be detected in separate PID elements further reduces the background of the $\eta \rightarrow \gamma \gamma$ decay with a subsequent $\gamma \rightarrow e^{+} e^{-}$conversion. In the higher dilepton mass bins, there is a contribution from decays of $\eta$ containing charged pions. These can be suppressed by cutting on variables describing the shape of the clusters in CB, such as number of crystals or effective radius vs the energy of the cluster.

The analysis yields $5.4 \times 10^{4} \eta$ Dalitz decays in total from both datasets. The resulting TFF distributions is fitted with (2) to extract the slope parameter $\Lambda^{-2}=\left(1.97 \pm 0.11_{t o t}\right) \mathrm{GeV}^{-2}$. The result is in excellent agreement with previous measurements and theoretical predictions.

\subsection{3 $\omega \rightarrow e^{+} e^{-} \pi^{0}$}

The same datasets used for the $\eta$ TFF study presented above, was also used to study the TFF from the $\omega \rightarrow \pi^{0} e^{+} e^{-}$decay [23]. This TFF has previously been measured by the NA60 collaboration $[24,25]$, with dimuons in the final state. The NA60 results show a puzzling discrepancy between theory and experiment.

In the analysis of the A2 data, events with exactly 5 clusters in the calorimeters are selected. The chance of a proton being unmeasured in this channel is very low, between 2.7$7.6 \%$, and allowing for unmeasured protons yields a much higher background. The leptons are identified in PID and can be separated from protons using their corresponding energy deposition. The kinematic fit procedure with the constraint of 4-momentum conservation for the signal channel was used. A significant background comes from $\omega \rightarrow \pi^{+} \pi^{-} \pi^{0}$. It is suppressed by performing a kinetic fit constrained by 4-momentum conservation for the signal channel and by the requirement of the $\pi^{0}$ mass as invariant mass of the two photons. Such a fit will yield a low fit probability for $\omega \rightarrow \pi^{+} \pi^{-} \pi^{0}$ events as well as shift the vertex position downstream. Cuts on these variables are then applied. Additional rejection of this background is achieved by cuts on the cluster shapes in the calorimeter. After the final selection, $\sim 1.1 \times 10^{3} \omega \rightarrow e^{+} e^{-} \pi^{0}$ events are used to create the TFF distributions. The resulting TFF data points are systematically lower than the NA60 result in the higher dilepton mass region, and hence more in agreement with recent theoretical predictions. However, the error bars, which are dominated by statistics, are overlapping with both NA60 data points and the theory predictions. Fitting the slope parametrisation (2) yields $\Lambda^{-2}=\left(1.99 \pm 0.21_{t o t}\right) \mathrm{GeV}^{-2}$. 


\subsection{4 $\eta^{\prime} \rightarrow e^{+} e^{-} \gamma$}

Due to the higher mass of the $\eta^{\prime}$, the available phase space for the dileptons goes slightly beyond the $\rho$ and $\omega$ pole regions. Hence, a precise measurement of this TFF allows for a clearer picture of the contributions of the vector mesons. There have been two previous measurements, one by Lepton-G [6] and one by BESIII [26].

In 2014, after installing the EPT, the A2 collaboration performed a dedicated measurement of $\eta^{\prime}$ [27]. During nearly 10 weeks of data taking, approximately 6 million $\eta^{\prime}$ were produced. One of the aims within this campaign is to measure the $\eta^{\prime} \rightarrow e^{+} e^{-} \gamma$ decay with the goal of extracting the TFF. Based on the estimated signal acceptance, it is projected that the A2 results will reach the $\rho$ pole.

\subsection{Dalitz plot studies}

\section{$3.3 \eta \rightarrow \pi^{0} \pi^{0} \pi^{0}$}

The A2 collaboration has published several measurements of the $\eta \rightarrow 3 \pi^{0}$ decay. The most recent one [28] is based on the same datasets used for the $\eta \rightarrow e^{+} e^{-} \gamma$ study presented above.

In the analysis, the $3 \pi^{0} p \rightarrow 6 \gamma p$ final state is selected by choosing events with 6 or 7 clusters in the calorimeters, allowing the proton to be unmeasured. A kinematic fit procedure requiring conservation of 4-momentum as well as $\pi^{0}$ mass constraints is used to select the most probable combinations of the photons and to reject background. The main source of background comes from $3 \pi^{0}$ events without an intermediate $\eta$. This background is kept at a sub-percent level by cuts on the kinematic fit probability and rejecting events with photon beam energies above $1.3 \mathrm{GeV}$.

The analysis yields $\sim 7 \times 10^{6} \eta \rightarrow 3 \pi^{0}$ events to be used to produce a Dalitz plot. The parametrisation given in (6) was fitted to the acceptance corrected Dalitz plot given by both only a z-distribution and a sextant $30^{\circ}<\phi<90^{\circ}$. Systematic checks evaluate the impact of the experimental resolution and boundary bins of the sextant as well as the two different data sets. The result shows a clear improvement of the goodness of fit when including the $\beta$ parameter. Additional improvement is achieved when also including a parametrisation of the cusp term, $2 \delta \sum_{i=1}^{3} \operatorname{Re} \sqrt{1-s_{i} / 4 m_{\pi^{ \pm}}^{2}}$, where $s_{i}=\left(P_{\eta}-P_{\pi_{i}}\right)^{2}$. Additionally, tests of the predictions by the NREFT framework was performed. The most recent A2 result yields the currently most precise measurement of the $\alpha$ parameter and a first measurement of the $\beta$ parameter as well as an indication of the cusp effect.

\section{$3.4 \eta^{\prime} \rightarrow \eta \pi^{0} \pi^{0}$}

The A2 collaboration has published a high statistics measurement of the $\eta^{\prime} \rightarrow \eta \pi^{0} \pi^{0}$ Dalitz plot [29] using the EPT data set. The final state $\pi^{0} \pi^{0} \eta p \rightarrow 6 \gamma p$ is selected by choosing events with 7 clusters in the calorimeters. To pair up the photons and reduce background, a kinematic fit procedure requiring 4-momentum conservation and mass constraints for $\pi^{0}$ and $\eta$ is applied. The analysis yields $\sim 1.13 \times 10^{5}$ signal events used to fill the Dalitz plot. The bins that crosses the kinematical border are not used. Bin-wise efficiency correction is applied. An independent analysis is performed as a cross-check, with one main difference being that the $\eta^{\prime}$ mass is used as a constraint in the kinematic fit. The two analysis strategies yield good agreement between the resulting Dalitz plots and $m_{\pi \pi}$ distributions. The resulting experimental Dalitz plot distribution is fitted by the parametrisation (5) . Additionally, the decay amplitude calculated within the NREFT framework is fitted to the experimental Dalitz 
plots. The fit yields a $\chi^{2} /$ d.o.f. of same magnitude as the standard fit, but provides a much better description of the $Y$ and $m_{\pi^{0} \pi^{0}}$ distribution in the cusp region. Additional fits are performed by allowing the $a_{0}-a_{2}$ to be a free parameter of the fit as well, and the outcome is in agreement with previously determined values. Hence, this study has provides the first observation of the cusp structure in the $\eta^{\prime} \rightarrow \eta \pi^{0} \pi^{0}$ channel.

\section{Summary}

With the high yield of photo-produced mesons, the A2 setup at MAMI is an ideal facility to perform precision measurements of decays of light mesons. The A2 collaboration has published results on TFF measurements from studies of $\pi^{0} / \eta \rightarrow e^{+} e^{-} \gamma$ as well as $\omega \rightarrow$ $e^{+} e^{-} \pi^{0}$. Additionally, A2 has recently published results of Dalitz plot studies of the decays $\eta \rightarrow 3 \pi^{0}$ and $\eta^{\prime} \rightarrow \eta \pi^{0} \pi^{0}$ with world leading accuracy. Ongoing projects within A2 include TFF measurements in the $\eta^{\prime} \rightarrow e^{+} e^{-} \gamma$ decay, as well as an even higher statistics result of the $\pi^{0}$ channel.

\section{References}

[1] G.W. Bennett et al. (Muon g-2), Phys. Rev. D 73, 072003 (2006)

[2] A. Keshavarzi, D. Nomura, T. Teubner, Phys. Rev. D97, 114025 (2018)

[3] B. Lee Roberts (Fermilab P989), Nucl. Phys. Proc. Suppl. 218, 237 (2011)

[4] H. Iinuma (J-PARC muon g-2/EDM), J. Phys. Conf. Ser. 295, 012032 (2011)

[5] I. Danilkin, C.F. Redmer, M. Vanderhaeghen (2019), arXiv: 1901.10346 [hep-ph]

[6] L.G. Landsberg, Phys. Rept. 128, 301 (1985)

[7] G. Colangelo, S. Lanz, H. Leutwyler, E. Passemar, Eur. Phys. J. C 78, 947 (2018)

[8] R. Escribano, P. Masjuan, J.J. Sanz-Cillero, JHEP 05, 094 (2011)

[9] S. Gonzàlez-Solís, E. Passemar, Eur. Phys. J. C 78, 758 (2018)

[10] T. Isken, B. Kubis, S.P. Schneider, P. Stoffer, Eur. Phys. J. C 77, 489 (2017)

[11] C.O. Gullstrom, A. Kupsc, A. Rusetsky, Phys. Rev. C 79, 028201 (2009)

[12] S.P. Schneider, B. Kubis, PoS CD09, 120 (2009)

[13] H. Herminghaus et al., IEEE Trans. Nucl. Sci. 30, 3274 (1983)

[14] K.H. Kaiser et al., Nucl. Instrum. Meth. A 593, 159 (2008)

[15] J.C. McGeorge et al., Eur. Phys. J. A 37, 129 (2008)

[16] P. Adlarson et al. (A2), Phys. Rev. C 92, 024617 (2015)

[17] S. Schumann et al., Eur. Phys. J. A 43, 269 (2010)

[18] P. Adlarson et al. (A2), Phys. Rev. C 95, 025202 (2017)

[19] T. Husek, K. Kampf, J. Novotny, Phys. Rev. D 92, 054027 (2015)

[20] C. Lazzeroni et al. (NA62), Phys. Lett. B 768, 38 (2017)

[21] H. Berghauser et al., Phys. Lett. B 701, 562 (2011)

[22] P. Aguar-Bartolome et al. (A2), Phys. Rev. C 89, 044608 (2014)

[23] P. Adlarson et al. (A2), Phys. Rev. C 95, 035208 (2017)

[24] R. Arnaldi et al. (NA60), Phys. Lett. B 677, 260 (2009)

[25] R. Arnaldi et al. (NA60), Phys. Lett. B 757, 437 (2016)

[26] M. Ablikim et al. (BESIII), Phys. Rev. D 92, 012001 (2015)

[27] O. Steffen (A2), EPJ Web Conf. 142, 01027 (2017)

[28] S. Prakhov et al. (A2), Phys. Rev. C 97, 065203 (2018)

[29] P. Adlarson et al. (A2), Phys. Rev. D 98, 012001 (2018) 